\section{Multimechanism approach benefits treatment of migraine}

A prospective multicenter study-one of the first of its kind-has shown encouraging results for a combined acute treatment for migraine, involving a triptan and an analgesic.

Smith et al. treated a cohort of 972 patients, containing both males and females, in their four-arm, double-blind study. The patients were randomly assigned to treatment with placebo, naproxen sodium $500 \mathrm{mg}$, sumatriptan $50 \mathrm{mg}$, or a combination of the two drugs at the aforementioned doses. At the onset of a moderate or severe migraine attack, patients completed study diary cards before taking their assigned medication. They then completed diary cards at $15 \mathrm{~min}$ intervals for up to 2 hours, and at $30 \mathrm{~min}$ intervals 2-4 hours after medication.

The primary endpoint of the study was a 'sustained pain response'. This was achieved in $46 \%$ of patients assigned to the combination therapy-significantly greater than that achieved in the patients taking sumatriptan (29\%), naproxen sodium (25\%), or placebo $(17 \%)(P<0.001)$. Headache response after 2 hours was also significantly higher in patients treated with combined medication than in those treated with sumatriptan, naproxen sodium, or placebo (65\% vs $49 \%, 46 \%$ and $27 \%$, respectively; $P<0.001$ ).

The authors suggest that multimechanism acute treatment for migraine using a triptan and an analgesic offers improved clinical benefits over monotherapy with these medications, and is well tolerated and not associated with a significant increase in adverse events compared with monotherapy.

Christine Kyme

Original article Smith TR et al. (2005) Sumatriptan and naproxen sodium for the acute treatment of migraine. Headache 45: 983-991

\section{Cognitive decline after CABG related to underlying cerebrovascular disease}

Coronary artery bypass grafting (CABG) is commonly thought to cause a decline in the postoperative cognitive functioning of patients. McKhann and colleagues, however, have recently reported results of a study that casts doubt on this claim.
The four-arm, prospective study investigated CABG patients $(n=140)$, patients undergoing off-pump coronary surgery $(n=72)$, nonsurgical cardiac controls with diagnosed coronary artery disease $(n=99)$, and hearthealthy controls with no cardiac risk factors $(n=69)$. Participants underwent a series of neuropsychological tests at baseline (before surgery), 3 months and 12 months. Scores from the tests were combined to form eight cognitive domain scores for cognitive abilities such as verbal memory and psychomotor speed.

Baseline performances of heart-healthy controls were found to be higher than those of the other three groups for all cognitive domains. For six of the domains, the difference between the performances of the healthy controls and the two surgical groups was significant $(P<0.01)$. All groups showed a similar degree of improvement in intrasubject scores from baseline to 3 months, and from 3 to 12 months. At 12 months all groups were performing at or above their baseline levels.

Concluding that there was no evidence that CABG patients' cognitive performance was worse than that of control patients with coronary artery disease over a 12-month followup period, the authors suggest that, in general, patients with long-standing coronary artery disease have an element of cognitive impairment caused by cerebrovascular disease, and that this is present before they undergo surgery.

Christine Kyme

Original article McKhann GM et al. (2005) Is there cognitive decline 1 year after CABG? Neurology 65: 991-999

\section{Novel target for anti-epileptics}

A recent US study carried out in rodents has shown that epileptic seizures can be triggered by glial as well as neuronal cell activity. This finding could have important implications for the future development of antiepileptic drugs.

Tian et al. carried out a series of electrophysiological experiments designed to characterize the cellular basis of hypersynchronous neuronal firing, one of the hallmarks of epilepsy. The researchers studied paroxysmal depolarization shifts (PDSs), and used combinations of sodium channel blockers and voltagegated calcium $\left(\mathrm{Ca}^{2+}\right)$ channel blockers to suppress synaptic activity. Unexpectedly, they 